\title{
A NUMERICAL TREATMENT OF A SUPERDEGENERATE EQUATION WITH APPLICATIONS TO THE POROUS MEDIA EQUATION
}

\author{
BY
}

M. BERTSCH (Università di Torino, Turin, Italy)

AND

R. DAL PASSO (Instituto per le Applicazioni del Calcolo, Rome, Italy)

1. Introduction. Consider the nonlinear diffusion problem

$$
\text { (I) } \begin{cases}u_{t}=\left(u^{m}\right)_{y y} & y \in \mathbb{R}, t>0 \\ u(y, 0)=u_{0}(y) & y \in \mathbb{R},\end{cases}
$$

where $m>1$ is a constant and where $u_{0}^{m-1}$ is a Lipschitz continuous nonnegative function. We shall assume that for some $a<b$

$$
u_{0}(y) \begin{cases}>0 & \text { if } a<y<b \\ =0 & \text { otherwise. }\end{cases}
$$

Equation (1.1) is called the porous media equation and Problem (I) has been studied extensively in the literature. In particular Problem (I) has a unique weak solution $u(y, t)$, which has compact support for all $t>0$, i.e., there exist Lipschitz continuous functions $\zeta_{i}:[0, \infty) \rightarrow \mathbb{R}(i=1,2)$ such that

$$
u(y, t) \begin{cases}>0 & \text { if } \zeta_{1}(t)<y<\zeta_{2}(t) \\ =0 & \text { otherwise. }\end{cases}
$$

The curves $y=\zeta_{i}(t)$ are called the interfaces or free boundaries, and there exist numbers $t_{i}^{*} \geq 0$, the so-called waiting-times, such that $\zeta_{i} \in C^{\infty}\left(\mathbb{R}^{+} \backslash t_{i}^{*}\right)$ and

$$
(-1)^{i} \zeta_{i}^{\prime}(t) \begin{cases}=0 & \text { if } 0<t<t_{i}^{*} \\ >0 & \text { if } t>t_{i}^{*} .\end{cases}
$$

It turns out that the differentiability of $\zeta_{i}$ at $t=t_{i}^{*}$ depends on the initial function $u_{0}$. For more precise and more general results we refer to the review papers by Aronson $[1,2]$, which also contain many references to the literature.

Several methods were proposed to give a numerical approximation of the solution $u$ of Problem (I) and its two interfaces $\zeta_{i}$. In some earlier papers, by Baklanovskaya [5], Berger, Brézis, and Rogers [6], Socolovsky [22], Graveleau and Jamet [13], and Rose $[18,19,20]$ only the approximation of $u$ itself was considered.

In more recent papers also the numerical construction of $\zeta_{i}$ has been studied. We can distinguish two different approaches. The first one is to solve a discretized version Received November 3, 1988.

(C)1990 Brown University 
of the free boundary problem for $u(y, t)$ and $\zeta_{i}(t)$. Here $\zeta_{1}(t)<y<\zeta_{2}(t)$ and the interface conditions are given by

$$
u\left(\zeta_{i}(t), t\right)=0
$$

and

$$
\zeta_{i}^{\prime}(t)=-\frac{m}{m-1}\left(u^{m-1}\right)_{y}\left(\zeta_{i}(t), t\right)
$$

This approach was chosen by Mimura, Nakaki, and Tomoeda [16] (see also [17]) and by DiBenedetto and Hoff [12] (see also [15]).

The second possibility is to find a transformation such that Problem (I) can be rewritten as an initial-boundary value problem, i.e., the free boundaries are replaced by fixed ones. Both Berryman [7] and Gurtin, MacCamy, and Socolovsky [14] propose methods based on such a transformation.

In the present paper we reconsider Berryman's transformation, which is based on an idea by Rosen [21]. Defining

$$
x=\int_{-\infty}^{y} u(s, t) d s, \quad L=\int_{a}^{b} u_{0}(s) d s,
$$

a straightforward calculation yields that $\tilde{u}(x, t)=u(y, t)$ satisfies the equation

$$
u_{t}=u^{2}\left(u^{m}\right)_{x x} \quad 0<x<L, t>0 .
$$

Let $C_{m}=m^{m /(m+1)}$ and define

$$
v(x, t)=C_{m} u^{m}(y, t), \quad v_{0}(x)=C_{m} u_{0}^{m}(y) .
$$

Then we arrive at the initial-boundary value problem

where

$$
\text { (II) } \begin{cases}v_{t}=v^{\alpha} v_{x x} & 0<x<L, t>0 \\ v(0, t)=v(L, t)=0 & t>0 \\ v(x, 0)=v_{0}(x) & 0<x<L,\end{cases}
$$

$$
\alpha=1+\frac{1}{m}, \quad \text { i.e., } 1<\alpha<2 .
$$

At this point Berryman proposes a method to solve Problem (II) numerically and he claims that the desired approximations of the interfaces can be obtained from the inverse transformation

$$
y(x, t)=y(x, 0)-C_{m}^{-1} \int_{0}^{t} v_{x}(x, s) d s,
$$

applied to $x=0$, and $x=L$, respectively.

In the above argument it is assumed that the solution $v(x, t)$ of Problem (II) is unique. However, as has been shown by Dal Passo and Luckhaus [11] in the case $\alpha=1$, Problem (II) possesses infinitely many solutions, and this nonuniqueness phenomenon carries over at once to the case $\alpha>1$ (for a general discussion of the nonuniqueness we refer to [8]; see also [23]). Observe that the nonuniqueness is due to the fact that, if $\alpha \geq 1$, the degeneracy of Eq. (1.5) is stronger than, for example, in the case of the porous media equation. Because of this we call Eq. (1.5) superdegenerate. 
It is the purpose of this paper to overcome this difficulty. In Sec. 2 we shall show that, if $v_{0}>0$ in $(0, L)$, Problem (II) has at most one solution $v$ which satisfies

$$
v(x, t)>0 \text { if } 0<x<L, t \geq 0 .
$$

From the transformation which we described above, it is clear that this is the solution which we try to approximate.

In Sec. 3 we describe a linearly implicit finite difference scheme to construct approximate solutions of Problem (II), which we denote by $v^{h}(x, t)$. The same type of scheme has also been used by Hoff in the case of the porous media equation. We shall prove that $v^{h} \rightarrow v$ uniformly as $h \rightarrow 0$, where $v$ is the unique positive solution of Problem (II).

We use (1.7) to construct the approximate interfaces $\zeta_{i}^{h}(t)$. In Secs. 4 and 5 we shall show that

$$
\zeta_{i}^{h} \rightarrow \zeta_{i} \quad \text { uniformly on bounded intervals as } h \rightarrow 0
$$

and

$$
\left(\zeta_{i}^{h}\right)^{\prime} \rightarrow \zeta_{i}^{\prime} \quad \text { uniformly on compact subsets of } \mathbb{R}^{+} \backslash t_{i}^{*} .
$$

In addition we prove a discretized version of the estimate

$$
(-1)^{i}\left\{\zeta_{i}^{\prime \prime}+\frac{m}{(m+1) t} \zeta_{i}^{\prime}\right\} \geq 0 \text { in } \mathscr{D}^{\prime}\left(\mathbb{R}^{+}\right)
$$

for $\zeta_{i}^{h}$ and we obtain (1.8) itself in the limit $h \rightarrow 0$. Estimate (1.8) was proved by Caffarelli and Friedman [10] and Vazquez [24] and has played a crucial role in the study of regularity properties of $\zeta_{i}$ [2]. The idea of our proof is different and can be used to prove similar estimates for more general equations (see [9]).

Finally, in Sec. 6, we discuss some computational results.

2. Uniqueness of the positive solution. First we collect some definitions and basic results about Problems (I) and (II). Here we shall assume that the initial functions $u_{0}$ and $v_{0}$ respectively satisfy the hypotheses

A1. $u_{0} \in C(\mathbf{R})$ satisfies $(1.2)$ and $\left(u_{0}^{m-1}\right)$ is Lipschitz continuous.

H1. $v_{0}$ is a Lipschitz continuous function on $[0, L]$ with Lipschitz constant $\gamma$, $v_{0}(0)=v_{0}(L)=0$, and $0<v_{0} \leq M$ in $(0 . L)$.

Definition 2.1. A solution of Problem (I) is a bounded nonnegative function $u \in C\left(\mathbf{R} \times \overline{\mathbf{R}}^{+}\right)$which satisfies

$$
\int_{\mathbf{R}} u(T) \psi(T)-\int_{\mathbf{R}} u_{0} \psi(0)=\int_{0}^{T} \int_{\mathbf{R}}\left\{u \psi_{t}+u^{m} \psi_{y y}\right\}
$$

for all $T>0$ and all functions $\psi \in C^{2,1}\left(\mathbf{R} \times \overline{\mathbb{R}^{+}}\right)$which have compact support.

Proposition 2.2. Let $u_{0}$ satisfy A1. Then Problem (I) has a unique solution.

For the proof (under less restrictive conditions on $u_{0}$ ) we refer to the literature mentioned in $[1,2]$.

Definition 2.3. A solution of Problem (II) is a nonnegative function $v \in$ $C([0, L] \times[0, \infty])$ such that for all $T>0$

(i) $v \in L^{\infty}\left((0, T) ; W^{1, \infty}(0, L)\right)$; 
(ii) for any $\psi \in C^{1,1}([0, L] \times[0, T])$ with $\psi(0, t)=\psi(L, t)=0$ if $0 \leq t \leq T$, $v$ satisfies

$$
\int_{0}^{L} v(T) \psi(T)-\int_{0}^{L} v_{0} \psi(0)=\int_{0}^{T} \int_{0}^{L}\left\{v \psi_{t}-v^{\alpha} v_{x} \psi_{x}-\alpha v^{\alpha-1} v_{x}^{2} \psi\right\} .
$$

The main result of this section is that Problem (II) has a unique positive solution. Theorem 2.4. Let $v_{0}$ satisfy hypothesis H1. Let $\alpha \geq 1$. Then Problem (II) possesses one and only one solution which satisfies

$$
v>0 \text { in }(0, L) \times[0, \infty) .
$$

Proof. It follows as in [23] that Problem (II) has a maximal solution, which we denote by $v(x, t)$, i.e., any other solution $w(x, t)$ satisfies $w \leq v$ in $[0, L] \times \mathbb{R}^{+}$(the construction of $v$ is straightforward: it is defined as the limit of a decreasing sequence of classical solutions $v_{n}(x, t)$ of Eq. (1.5) which are strictly positive in $[0, L] \times \mathbb{R}^{+}$; by the maximum principle $w \leq v_{n}$ for all $n$ and thus $w \leq v$; the positivity of $v$ follows for example from comparison with explicit subsolutions).

It remains to prove the uniqueness. So let $w$ be a second positive solution of Problem (II). Since $v \geq w>0$ in $(0, L) \times \mathbf{R}^{+}, v$ and $w$ are both classical solutions of Eq. (1.5). We argue by contradiction and suppose that for some $T>0, w \not \equiv v$ in $(0, L) \times(0, T)$.

Since $w \leq v$, it follows that the function

$$
g(x)=\int_{0}^{T}(v-w)(x, s) d s
$$

attains a positive maximum in $(0, L)$, say at $x=x_{0}$. Thus

$$
g\left(x_{0}\right)>0
$$

and

$$
g^{\prime}\left(x_{0}\right)=\int_{0}^{T}(v-w)_{x}\left(x_{0}, s\right) d s=0 .
$$

Integrating Eq. (1.5) it follows that for all $x_{0}<y<L$ and $0<\tau<T$

$$
\left.\int_{x_{0}}^{y} \frac{1}{1-\alpha}\left(v^{1-\alpha}-w^{1-\alpha}\right)\right|_{t=\tau} ^{t=T}=\left.\int_{\tau}^{T}(v-w)_{x}\right|_{x=x_{0}} ^{x=y}
$$

(here we assume that $\alpha>1$; if $\alpha=1$ the proof is similar). If $\tau \searrow 0$, we obtain that

$$
g^{\prime}(y)=g^{\prime}\left(x_{0}\right)+\int_{x_{0}}^{y} \frac{1}{1-\alpha}\left(v^{1-\alpha}-w^{1-\alpha}\right)(s, T) d s \geq 0 .
$$

Hence $g$ is nondecreasing on $\left(x_{0}, L\right)$ and thus $g(L) \geq g\left(x_{0}\right)>0$. Since $g(L)=0$ we have found a contradiction.

REMARK. Hypothesis $\mathrm{H} 1$ can be relaxed. In particular the Lipschitz condition is not necessary. 
3. Problem $\left(\mathrm{II}_{\mathrm{h}}\right)$. In this section we shall construct approximate solutions of Problem (II) which converge to the unique positive solution $v(x, t)$.

Let $T>0$ and let $N$ be a positive integer. We set

$$
\Delta t=\frac{T}{N} \quad \text { and } \quad t_{n}=n \Delta t \text { for } n=0,1, \ldots, N,
$$

and for every $N$ we choose a positive integer $K$ and points

$$
0=x_{0}<x_{1}<x_{2}<\cdots<x_{K}=L .
$$

Let

$$
\Delta x_{k}=x_{k}-x_{k-1} \text { for } k=1, \ldots, K,
$$

and

$$
\Delta x=\max \left\{\Delta x_{k}, k=1,2, \ldots, K\right\},
$$

and we define the vector $h=(\Delta x, \Delta t)$. We shall always assume that $\Delta x \rightarrow 0$ as $\Delta t \rightarrow 0$.

We consider the discretized problem

$$
\left(\mathrm{II}_{\mathrm{h}}\right) \begin{cases}\frac{v_{k}^{n+1}-v_{k}^{n}}{\Delta t}=\left(v_{k}^{n}\right)^{\alpha} A v_{k}^{n+1} & k=1, \ldots, K-1 ; n=0, \ldots, N-1 \\ v_{0}^{n}=v_{K}^{n}=0 & n=0, \ldots, N \\ v_{k}^{0}=v_{0}\left(x_{k}\right) & k=0, \ldots, K,\end{cases}
$$

with $\alpha \geq 1$ and

$$
\begin{aligned}
A v_{k}^{n} & =2\left\{\frac{v_{k+1}^{n}}{\Delta x_{k+1}\left(\Delta x_{k}+\Delta x_{k+1}\right)}-\frac{v_{k}^{n}}{\Delta x_{k} \Delta x_{k+1}}+\frac{v_{k-1}^{n}}{\Delta x_{k}\left(\Delta x_{k}+\Delta x_{k+1}\right)}\right\} \\
& =\frac{2}{\Delta x_{k}+\Delta x_{k+1}}\left(w_{k+1}^{n}-w_{k}^{n}\right)
\end{aligned}
$$

where $w_{k}^{n}$ is the discretized spatial derivative

$$
w_{k}^{n}=\frac{v_{k}^{n}-v_{k-1}^{n}}{\Delta x_{k}} .
$$

Problem $\left(\mathrm{II}_{\mathrm{h}}\right)$ is a linearly implicit finite difference scheme. A similar scheme was used by Hoff [15] to discretize the porous media equation.

First we collect some basic results about Problem $\left(\mathrm{II}_{\mathrm{h}}\right)$.

Proposition 3.1. Let $v_{0}$ satisfy hypothesis $\mathrm{H} 1$ and let $\alpha \geq 1$. Then Problem $\left(\mathrm{II}_{\mathrm{h}}\right)$ has a unique solution $\left\{v_{k}^{n}\right\}_{k=0, \ldots, K}^{n=0, \ldots, N}$ which satisfies the following properties

(i) $0 \leq v_{k}^{n} \leq M=\max \left\{v_{0}(x) ; 0 \leq x \leq L\right\}$;

(ii) $\left|w_{k}^{n}\right| \leq \gamma$;

(iii) there exists a constant $\mathscr{C}$ which only depends on $M$ and $\gamma$ such that

$$
\left|v_{k}^{n}-v_{k}^{m}\right| \leq \mathscr{C}\left\{\left|t_{n}-t_{m}\right|^{1 / 2}+\Delta x\right\}
$$

for all $t_{n}, t_{m} \in[0, T]$ and all $k$, where $\Delta x$ is defined by (3.1).

The proofs are similar to the ones given by Hoff [15]. In particular (i) and (ii) follow easily from an induction argument. Here we shall only give the proof of (iii). 
Proof of (iii). First we observe that, if we define for example $\Delta x_{k}=\Delta x$ for all $k<1$ and $k>K$, and if we extend the solution by $v_{k}^{n}=0$ for $k<0$ and $k>K, v_{k}^{n}$ satisfies the scheme for all integers $k$.

Let $k_{0}$ be an integer and define

$$
H=\max _{n_{0} \leq n \leq n_{1}}\left|v_{k_{0}}^{n}-v_{k_{0}}^{n_{0}}\right| .
$$

Let $\rho \geq 2 \Delta x$ to be chosen below and define

$$
V_{k}^{n}=v_{k}^{n}-v_{k_{0}}^{n_{0}}-\gamma \rho-\frac{H}{\rho^{2}}\left\{4\left(x_{k}-x_{0}\right)^{2}+c\left(t_{n}-t_{n_{0}}\right)\right\} .
$$

If we choose $c=8 M^{\alpha}$ we obtain that

$$
V_{k}^{n+1}-V_{k}^{n}-\left(v_{k}^{n}\right)^{\alpha} \Delta t A V_{k}^{n+1}=\left\{-\frac{H c}{\rho^{2}}+8\left(v_{k}^{n}\right)^{\alpha} \frac{H}{\rho^{2}}\right\} \Delta t \leq 0 .
$$

We claim that

$$
V_{k}^{n} \leq 0 \text { if } n_{0} \leq n \leq n_{1} \text { and }\left|x_{k}-x_{k_{0}}\right| \leq \rho .
$$

For $n=n_{0}$, (3.3) follows at once. Assume that (3.3) holds for some $n$, and choose $k$ such that $V_{k}^{n+1}$ is maximal with respect to $k$. If $\left|x_{k \pm 1}-x_{k_{0}}\right| \leq \rho$, we obtain from (3.2) that $V_{k}^{n+1} \leq 0$. If $\left|x_{k+1}-x_{k_{0}}\right|>\rho$ or $\left|x_{k-1}-x_{k_{0}}\right|>\rho$, then $\left|x_{k}-x_{k_{0}}\right| \geq \rho-\Delta x \geq \frac{1}{2} \rho$ and hence

$$
V_{k}^{n+1} \leq v_{k}^{n+1}-v_{k_{0}}^{n+1}-\gamma \rho+v_{k_{0}}^{n+1}-v_{k_{0}}^{n_{0}}-H \leq 0 .
$$

Thus $V_{k}^{n+1} \leq 0$ if $\left|x_{k}-x_{k_{0}}\right| \leq \rho$ and (3.3) follows.

We derive from (3.3) that

$$
v_{k_{0}}^{n}-v_{k_{0}}^{n_{0}} \leq \gamma \rho+\frac{H c}{\rho^{2}}\left(t_{n_{1}}-t_{n_{0}}\right) \quad \text { if } n_{0} \leq n \leq n_{1} .
$$

The same inequality can be proved for $v_{k_{0}}^{n_{0}}-v_{k_{0}}^{n}$. Hence, maximizing over $n$, we find that

$$
H \leq \gamma \rho+\frac{c\left(t_{n_{1}}-t_{n_{0}}\right)}{\rho^{2}} H .
$$

Choosing $\rho_{1}+2 \Delta x \leq \rho<\rho_{1}+3 \Delta x$, where

$$
\rho_{1}=\left\{2 c\left(t_{n_{1}}-t_{n_{0}}\right)\right\}^{1 / 2}
$$

we conclude that

$$
H \leq 2 \gamma \rho \leq \mathscr{C}(\gamma, M)\left\{\left(t_{n_{1}}-t_{n_{0}}\right)^{1 / 2}+\Delta x\right\},
$$

which completes the proof.

An important estimate is the following discretized version of the estimate

$$
v_{t} \geq-\frac{1}{\alpha t} v \quad \text { in } \mathscr{D}^{\prime}\left((0, L) \times \mathbb{R}^{+}\right)
$$

which is satisfied by the unique positive solution $v$ of Problem (II). First we introduce a condition on the mesh points: for all $1 \leq k \leq K-1$

$$
\frac{\min \left\{x_{k}^{\alpha-1},\left(L-x_{k}\right)^{\alpha-1}\right\} \Delta t}{\frac{1}{2}\left(\Delta x_{k}+\Delta x_{k+1}\right)} \leq \frac{1}{2 \alpha \gamma^{\alpha}} .
$$


Lemma 3.2. Let $v_{0}$ satisfy hypothesis $\mathrm{H} 1$ and let $\alpha \geq 1$. If (3.4) is satisfied, then

$$
\frac{v_{k}^{n+1}-v_{k}^{n}}{\Delta t} \geq-\frac{1}{\alpha t_{n+1}} v_{k}^{n} \quad k=0, \ldots, K ; 0 \leq n<N .
$$

Proof. We define

$$
z_{k}^{n}=\frac{v_{k}^{n+1}-v_{k}^{n}}{v_{k}^{n} \Delta t}=\left(v_{k}^{n}\right)^{\alpha-1} A v_{k}^{n+1} \quad 1 \leq k \leq K-1 ; 0 \leq n<N .
$$

We have to prove that

$$
z_{k}^{n} \geq-\frac{1}{\alpha t_{n}}
$$

We proceed by induction. Let $n=0$. Then, by Proposition 3.1 (ii) and condition (3.4),

$$
\begin{aligned}
\left|z_{k}^{0}\right| & =\left|\left(v_{k}^{0}\right)^{\alpha-1} A v_{k}^{1}\right|=\left|\left(v_{k}^{0}\right)^{\alpha-1} \frac{w_{k+1}^{1}-w_{k}^{1}}{\frac{1}{2}\left(\Delta x_{k}+\Delta x_{k+1}\right)}\right| \\
& \leq 2 \gamma \frac{\left(v_{k}^{0}\right)^{\alpha-1}}{\frac{1}{2}\left(\Delta x_{k}+\Delta x_{k+1}\right)} \leq 2 \gamma^{\alpha} \frac{\min \left\{x_{k}^{\alpha-1},\left(L-x_{k}\right)^{\alpha-1}\right\}}{\frac{1}{2}\left(\Delta x_{k}+\Delta x_{k+1}\right)} \\
& \leq \frac{1}{\alpha \Delta t}=\frac{1}{\alpha t_{1}}
\end{aligned}
$$

and (3.5) follows for $n=0$.

Let $n \geq 1$ and assume that (3.5) holds for $n-1$. Since

$$
v_{k}^{n+1}=v_{k}^{n}\left(1+z_{k}^{n} \Delta t\right)
$$

we find that

$$
z_{k}^{n}=\left(v_{k}^{n}\right)^{\alpha-1} A\left\{v_{k}^{n}\left(1+z_{k}^{n} \Delta t\right)\right\}=\Delta t\left(v_{k}^{n}\right)^{\alpha-1} A\left(v_{k}^{n} z_{k}^{n}\right)+\left(\frac{v_{k}^{n}}{v_{k}^{n-1}}\right)^{\alpha-1} z_{k}^{n-1} .
$$

Suppose that $z_{k}^{n}$ is minimal with respect to $k$. We evaluate

$$
\begin{aligned}
A\left(v_{k}^{n} z_{k}^{n}\right) & =\frac{2}{\Delta x_{k}+\Delta x_{k+1}}\left\{\frac{v_{k+1}^{n} z_{k+1}^{n}-v_{k}^{n} z_{k}^{n}}{\Delta x_{k+1}}+\frac{v_{k-1}^{n} z_{k-1}^{n}-v_{k}^{n} z_{k}^{n}}{\Delta x_{k}}\right\} \\
& \geq \frac{2 z_{k}^{n}}{\Delta x_{k}+\Delta x_{k+1}}\left\{\frac{v_{k+1}^{n}-v_{k}^{n}}{\Delta x_{k+1}}+\frac{v_{k-1}^{n}-v_{k}^{n}}{\Delta x_{k}}\right\} \\
& =z_{k}^{n}\left(v_{k}^{n-1}\right)^{1-\alpha} z_{k}^{n-1}
\end{aligned}
$$

and thus

$$
z_{k}^{n} \geq z_{k}^{n-1}\left(\frac{v_{k}^{n}}{v_{k}^{n-1}}\right)^{\alpha-1}\left(1+\Delta t z_{k}^{n}\right)=z_{k}^{n-1}\left(1+z_{k}^{n-1} \Delta t\right)^{\alpha-1}\left(1+z_{k}^{n} \Delta t\right) .
$$

Since the function $s \rightarrow s(1+s)^{\alpha-1}$ is increasing if $s>-\frac{1}{\alpha}$ and since $\Delta t z_{k}^{n-1} \geq-\frac{1}{\alpha n} \geq$ $-\frac{1}{\alpha}$, we obtain that

$$
z_{k}^{n} \geq-\frac{1}{\alpha n \Delta t}\left(1-\frac{1}{\alpha n}\right)^{\alpha-1}\left(1+z_{k}^{n} \Delta t\right)
$$


Hence

and because

$$
z_{k}^{n} \geq-\frac{\frac{1}{n}}{\left(1-\frac{1}{\alpha n}\right)^{1-\alpha}+\frac{1}{\alpha n}} \cdot \frac{1}{\alpha \Delta t}
$$

$$
\left(1-\frac{1}{\alpha n}\right)^{1-\alpha} \geq 1+(\alpha-1) \frac{1}{\alpha n}=1+\frac{1}{n}-\frac{1}{\alpha n}
$$

it follows that

$$
z_{k}^{n} \geq-\frac{1}{\alpha(n+1) \Delta t}=-\frac{1}{\alpha t_{n+1}}
$$

and the proof is complete.

We define the function $v^{h}$ on $[0, L] \times[0, T]$ by

$$
v^{h}(x, t)=v_{k}^{n}+\left(x-x_{k}\right) w_{k}^{n} \quad \text { if } x_{k} \leq x \leq x_{k+1}, t_{n} \leq t \leq t_{n+1} .
$$

Observe that, since $\left|w_{k}^{n}\right| \leq \gamma$, the spatial derivative $v_{x}^{h}$ belongs to $L^{\infty}((0, L) \times(0, T))$.

Theorem 3.3. Let $v_{0}$ satisfy hypothesis $\mathrm{H} 1$, let $\alpha \geq 1$, and let the mesh condition (3.4) be satisfied. Let $v$ denote the unique positive solution of Problem (II). Then

(i) $v^{h} \rightarrow v$ uniformly on $[0, L] \times[0, T]$ as $h \rightarrow 0$;

(ii) $v_{x}^{h} \rightarrow v_{x}$ uniformly on compact subsets of $(0, L) \times(0, T)$ as $h \rightarrow 0$.

Proof. By Proposition 3.1 there exist a subsequence $v^{h_{i}}$ and a function $v$ which is Lipschitz continuous in $x$ and Hölder continuous in $t$ (exponent $\frac{1}{2}$ ) such that $v^{h_{i}} \rightarrow v$ uniformly on $[0, L] \times[0, T]$ as $h_{i} \rightarrow 0$. For $0<x_{0}<L, v\left(x_{0}, 0\right)=v_{0}\left(x_{0}\right)>0$ and thus, by continuity of $v, v\left(x_{0}, t\right)>0$ for small values of $t$. In addition it follows from Lemma 3.2 that $v_{t} \geq-\frac{1}{\alpha t} v$ in the sense of distributions and hence $v\left(x_{0}, t\right)>0$ for all $t>0$. Thus

$$
v>0 \text { in }(0, L) \times[0, \infty) .
$$

By Lemma 3.2

$$
A v_{k}^{n+1} \geq-\frac{1}{\alpha t_{n+1}}\left(v_{k}^{n}\right)^{1-\alpha} .
$$

Writing $|a|=a+2 a^{-}(a \in \mathbf{R})$, this implies that

$$
\begin{aligned}
\sum_{k=k_{0}}^{k_{1}}\left|A v_{k}^{n}\right| \frac{1}{2}\left(\Delta x_{k}+\Delta x_{k+1}\right) & =\sum_{k=k_{0}}^{k_{1}}\left\{A v_{k}^{n}+2\left(A v_{k}^{n}\right)^{-}\right\} \frac{1}{2}\left(\Delta x_{k}+\Delta x_{k+1}\right) \\
& \leq w_{k_{1}+1}-w_{k_{0}}+\frac{1}{\alpha t_{n}} \sum_{k=k_{0}}^{k_{1}}\left(v_{k}^{n-1}\right)^{1-\alpha}\left(\Delta x_{k}+\Delta x_{k+1}\right) .
\end{aligned}
$$

If $0<\varepsilon \leq x_{k_{0}}<x_{k_{1}} \leq L-\varepsilon$ and $t \geq \varepsilon$, we find that for some $C(\varepsilon)$ which does not depend on $h$

$$
\sum_{k=k_{0}}^{k_{1}}\left|A v_{k}^{n}\right| \frac{1}{2}\left(\Delta x_{k}+\Delta x_{k+1}\right) \leq 2 \gamma+C(\varepsilon)
$$

Since $\left|v_{x}^{h}\right| \leq \gamma$, this implies (cf. [13]) that $v_{x}^{h_{i}} \rightarrow v_{x}$ in $L^{p}((\varepsilon, L-\varepsilon) \times(\varepsilon, T])$ as $h_{i} \rightarrow 0$, for all $1 \leq p<\infty$. Because $\varepsilon$ is arbitrary, $v_{x}^{h_{1}} \rightarrow v_{x}$ in $L^{2}((0, L) \times(0, T))$ and it 
follows at once that $v$ is a solution of Problem (II). By (3.6) and Theorem 2.4, $v$ is the unique positive solution of Problem (II), and $v^{h} \rightarrow v$ as $h \rightarrow 0$.

Finally, the second part of Theorem 3.3 follows easily from the continuity of $v_{x}$ and the fact that, by (3.6) and (3.7), $A v_{k}^{n}$ is uniformly bounded from below on compact subsets of $(0, L) \times(0, T)$.

4. Problem (I): the interface curves. The main purpose of this paper is to give a numerical construction of the interfaces $\zeta_{1}(t)$ and $\zeta_{2}(t)$, using the transformation of Problem (I) into Problem (II) as we explained in the introduction. In view of formula (1.7) it is natural to define the approximate interfaces by

$$
\begin{aligned}
& \zeta_{1}^{h}(t)=a-C_{m}^{-1} \int_{0}^{t} v_{x}^{h}(0, s) d s, \\
& \zeta_{2}^{h}(t)=b-C_{m}^{-1} \int_{0}^{t} v_{x}^{h}(L, s) d s,
\end{aligned}
$$

where $v^{h}$ is defined as in Theorem 3.3, with $\alpha=1+\frac{1}{m} \in(1,2)$ and $v_{0}$ defined by (1.4):

$$
v_{0}(x)=C_{m} u_{0}^{m}(y) .
$$

Before we discuss the convergence of $\zeta_{i}^{h}$ to $\zeta_{i}$ as $h \rightarrow 0$, we pay some attention to the construction of $v_{0}(x)$. In (4.2) we have assumed that, at the time $t=0$, the transformation from $y$ to $x$ is explicitly known from (1.3):

$$
x=\int_{a}^{y} u_{0}(s) d s .
$$

However, in most of the cases $x$ cannot be computed explicitly. To overcome this problem, we approximate $u_{0}^{m-1}$ by a piecewise linear function $\left(u_{0}^{m-1}\right)^{\tilde{h}}$, where $\left(u_{0}^{m-1}\right)^{\tilde{h}} \rightarrow\left(u_{0}^{m-1}\right)$ as $\tilde{h} \rightarrow 0$. This implies that $v_{0}$, and thus Problem $\left(\mathrm{II}_{\mathrm{h}}\right)$ as well as its solution $v^{h}$, depend on $\tilde{h}$ (in particular $L$ depends on $\tilde{h} !$ ).

In the rest of the paper we shall always suppress this dependence on $\tilde{h}$. In particular we shall always assume that $\tilde{h} \rightarrow 0$ as $h \rightarrow 0$. Mathematically this procedure is justified by the fact all the estimates which we have given in the previous section, and the ones which will follow below, hold uniformly with respect to $\tilde{h}$. The proofs are chosen in such a way that this can be easily checked by the interested reader. Finally we observe that, from a computational point of view, it is quite natural to assume that the error in the approximation of $u_{0}(y)$ is much smaller than the error which we make if we replace $v(x, t)$ by $v^{h}(x, t)$.

To prove the convergence of $\zeta_{i}^{h}$ to $\zeta_{i}$, we need an additional condition on the initial function.

A2. There exists an $\varepsilon>0$ such that either $u_{0}^{m-1}(y) \geq \mathscr{C}(y-a)$ on $[a, a+\varepsilon]$ (respectively, $u_{0}^{m-1}(y) \geq \mathscr{C}(b-y)$ on $[b-\varepsilon, b]$ ) for some $\mathscr{C}>0$ or $u_{0}^{m-1}$ is convex on $[a, a+\varepsilon]$ (respectively on $[b-\varepsilon, b]$ ). 
The following Lemma shows that this condition can be translated into a condition on the transformed initial function:

H2. $v_{0}^{1-\alpha} \in L^{1}(0, L)$, and there exists an $\varepsilon>0$ such that either $v_{0}(x) \geq \mathscr{C} x$ on $[0, \varepsilon]$ (respectively, $v_{0}(x) \geq \mathscr{C}(L-x)$ on $[L-\varepsilon, L]$ ) for some $\mathscr{C}>0$, or $v_{0}$ is convex on $[0, \varepsilon]$ (respectively on $[b-\varepsilon, b]$ ).

Lemma 4.1. Let $u_{0}$ satisfy hypotheses A $1-2$ and let $L, \alpha$ and $v_{0}$ be defined by (1.3), (1.6), and (4.2). Then $v_{0}$ satisfies H1-2.

Proof. We only have to check H2. By (1.3), (1.4), and (1.6), at $t=0$

$$
\frac{\partial y}{\partial x}=\frac{1}{u_{0}}=C v_{0}^{-1 / m}=C v_{0}^{1-\alpha}
$$

for some $C>0$, and thus

$$
C \int_{0}^{L} v_{0}^{1-\alpha}(x) d x=b-a<\infty .
$$

The rest of condition $\mathrm{H} 2$ follows from the relation

$$
\frac{d v_{0}}{d x}=\left.C_{m} \frac{d u_{0}^{m}}{d y} \cdot \frac{\partial y}{\partial x}\right|_{t=0}=\frac{m C_{m}}{m-1} \frac{d u_{0}^{m-1}}{d y}
$$

which holds for a.e. $x$ and $y$.

In order to state our main result, we have to introduce the additional mesh condition that for certain positive constants $A_{1}$ and $A_{2}$ which do not depend on $h$,

$$
A_{1} \leq \Delta x_{k+1} / \Delta x_{k} \leq A_{2} \quad(k=1, \ldots, K-1) .
$$

TheOREM 4.2. Let $u_{0}$ satisfy hypotheses A1-2 and let $L, \alpha$ and $v_{0}$ be defined by (1.3), (1.6), and (4.2). Let $T>0$, let the mesh conditions (3.4) and (4.3) be satisfied and let $v^{h}$ be the solution of Problem $\left(\mathrm{II}_{\mathrm{h}}\right)$. Let $\zeta_{i}(i=1,2)$ be the interfaces of Problem (I) and let $\zeta_{i}^{h}$ be defined by (4.1). Then, for $i=1,2$,

$$
\zeta_{i}^{h} \rightarrow \zeta_{i} \quad \text { uniformly on }[0, T] \text { as } h \rightarrow 0
$$

and

$$
\left(\zeta_{i}^{h}\right)^{\prime} \rightarrow \zeta_{i}^{\prime} \text { uniformly on compact subsets of }(0, T] \backslash t_{i}^{*} \text { as } h \rightarrow 0 .
$$

First we prove an auxiliary result about Problem (II).

Lemma 4.3. Let $v_{0}$ satisfy hypothesis $\mathrm{H} 1$ and let $v(x, t)$ be the unique positive solution of Problem (II) with $1<\alpha<2$. If

$$
\int_{0}^{L / 2} v_{0}^{1-\alpha}(x) d x<\infty
$$

then

$$
v_{x}(0, t)=\lim _{x \searrow 0} v_{x}(x, t)
$$

exists for all $t>0$.

REMARK. A similar result holds if $x=0$ is replaced by $x=L$.

Proof. Let $0<\varepsilon<L / 2$ and $0<\tau<t \leq T$. Then

$$
\int_{\tau}^{t} \int_{\varepsilon}^{L / 2}\left(v^{1-\alpha}\right)_{t}=-(\alpha-1) \int_{\tau}^{t} \int_{\varepsilon}^{L / 2} v_{x x}
$$


and thus

$$
\left.\int_{\varepsilon}^{L / 2} v^{1-\alpha}\right|_{\tau} ^{t}=-\left.(\alpha-1) \int_{\tau}^{t} v_{x}\right|_{x=\varepsilon} ^{x=L / 2}
$$

Letting $\tau \searrow 0$,

$$
\begin{aligned}
\int_{\varepsilon}^{L / 2} v^{1-\alpha}(t) & =\int_{\varepsilon}^{L / 2} v_{0}^{1-\alpha}-\left.(\alpha-1) \int_{0}^{t} v_{x}\right|_{x=\varepsilon} ^{x=L / 2} \\
& \leq \int_{0}^{L / 2} v_{0}^{1-\alpha}+2 \gamma(\alpha-1) T
\end{aligned}
$$

and we conclude that there exists a constant $C_{0}$ such that

$$
\int_{0}^{L / 2} v^{1-\alpha}(t) \leq C_{0} \quad \text { for } 0 \leq t \leq T
$$

Since (cf. (3.7))

$$
v_{x x}=v^{-\alpha} v_{t} \geq-\frac{1}{\alpha t} v^{1-\alpha} \quad \text { on }(0, L) \times \mathbb{R}^{+}
$$

it follows from (4.4) that for some $C_{1}$

$$
\int_{0}^{L / 2}\left|v_{x x}\right| d x=\int_{0}^{L / 2} v_{x x}+2 \int_{0}^{L / 2}\left\{v_{x x}\right\}^{-} \leq C_{1}\left(1+\frac{1}{t}\right)
$$

Hence, for $t>0$, the function $v_{x}(t)$ is absolutely continuous on $\left(0, \frac{1}{2} L\right)$ and Lemma 4.3 follows.

A consequence of Lemma 4.3 is that, by the Dominated Convergence Theorem,

$$
\int_{0}^{t}\left(v_{x}(\delta, s)-v_{x}(0, s) d s \rightarrow 0 \quad \text { as } \delta \searrow 0\right.
$$

uniformly on bounded intervals $[0, T]$. The crucial estimate, which we need in order to prove Theorem 4.2, is a discretized analogue of (4.5).

LEMMA 4.4. Let $v_{0}$ satisfy hypotheses $\mathrm{H} 1-2$, let $T>0$ and $1<\alpha<2$, let the mesh conditions (3.4) and (4.3) be satisfied, and let $\left\{v_{k}^{n}\right\}_{k=0, \ldots, K}^{n=0, \ldots, N}$ be the solution of Problem $\left(\mathrm{II}_{\mathrm{h}}\right)$. For all $\varepsilon>0$ there exist numbers $\delta>0$ and $h_{0}>0$ such that if $|h|<h_{0}$, then

$$
\left|\sum_{n=0}^{n_{1}}\left(w_{k_{0}}^{n}-w_{1}^{n}\right) \Delta t\right|<\varepsilon \quad \text { for } 0 \leq t_{n_{1}} \leq T, 0<x_{k_{0}}<\delta .
$$

We postpone the proof to the next section.

Proof of Theorem 4.2. We only give the proof for $i=1$.

First we show that

$$
I(t) \equiv \int_{0}^{t}\left\{\left(v^{h}\right)_{x}(0, s)-v_{x}(0, s)\right\} d s \rightarrow 0 \quad \text { uniformly on }[0, T] \text { as } h \rightarrow 0 .
$$

We set

$$
I(t)=I_{1}(t)+I_{2}(t)+I_{3}(t), \quad 0 \leq t \leq T
$$


where, for some $\delta>0$ to be chosen,

$$
\begin{aligned}
& I_{1}(t)=\int_{0}^{t}\left\{\left(v^{h}\right)_{x}(0, s)-\left(v^{h}\right)_{x}(\delta, s)\right\} d s \\
& I_{2}(t)=\int_{0}^{t}\left\{\left(v^{h}\right)_{x}(\delta, s)-v_{x}(\delta, s)\right\} d s \\
& I_{3}(t)=\int_{0}^{t}\left\{v_{x}(\delta, s)-v_{x}(0, s)\right\} d s .
\end{aligned}
$$

Let $\varepsilon>0$. By Lemma 4.1 we may apply Lemma 4.4 and the consequence (4.5) of Lemma 4.3. Hence, for $\delta>0$ and $|h|>0$ small enough,

$$
\left|I_{1}(t)\right|+\left|I_{3}(t)\right| \leq \frac{1}{2} \varepsilon
$$

So we choose $\delta>0$. Then, by Theorem 3.3 (ii), we can choose $|h|$ so small that also

$$
\left|I_{2}(t)\right| \leq \frac{1}{2} \varepsilon
$$

and (4.6) follows.

Since, by (1.7) and (4.1),

$$
\zeta_{1}^{h}(t)-\zeta_{1}(t)=-C_{m}^{-1} I(t)
$$

it follows at once from (4.6) that

$$
\zeta_{1}^{h} \rightarrow \zeta_{1} \quad \text { uniformly on }[0, T] \text { as } h \rightarrow 0 \text {. }
$$

By the definition of the waiting-time $t_{1}^{*}$

$$
v_{x}(0, t)=0 \text { for } 0<t<t_{1}^{*}
$$

(this follows easily, for example, from an argument by contradiction). In addition, if we translate a result by Caffarelli and Friedman [10] about the porous media equation to the solution $v(x, t)$, the function $v_{x}(0, t)$ is continuous at points $t>t_{1}^{*}$. Hence

$$
v_{x}(0, t) \quad \text { is continuous on } \mathbf{R}^{+} \backslash t_{1}^{*} \text {. }
$$

On the other hand, by Lemma 3.2 ,

$$
\begin{aligned}
\frac{v_{x}^{h}\left(0, t_{n}+\Delta t\right)-v_{x}^{h}\left(0, t_{n}\right)}{\Delta t} & =\frac{w_{1}^{n+1}-w_{1}^{n}}{\Delta t}=\frac{v_{1}^{n+1}-v_{1}^{n}}{\Delta x_{1} \Delta t} \\
& \geq-\frac{1}{\alpha t_{n+1}} \cdot \frac{v_{1}^{n}}{\Delta x_{1}}=-\frac{w_{1}^{n}}{\alpha t_{n+1}}=-\frac{1}{\alpha t_{n+1}} v_{x}^{h}\left(0, t_{n}\right) .
\end{aligned}
$$

If we combine this with (4.5) and (4.7) we obtain that

$$
v_{x}^{h}(0, t) \rightarrow v_{x}(0, t) \quad \text { as } h \rightarrow 0,
$$

uniformly on compact subsets of $\mathbf{R}^{+} \backslash t_{1}^{*}$, and thus the same holds for the convergence of $\left(\zeta_{1}^{h}\right)^{\prime}(t)$ to $\zeta_{1}^{\prime}(t)$. 
RemarKs. (i) Observe that (4.8) is the discretized version of the estimate

$$
-\zeta_{1}^{\prime \prime} \geq \frac{1}{\alpha t} \zeta_{1}^{\prime}=\frac{m}{(m+1) t} \zeta_{1}^{\prime} \quad \text { in } \mathscr{D}^{\prime}\left(\mathbf{R}^{+}\right)
$$

which was given by Caffarelli and Friedman [10] and Vazquez [24].

(ii) Studying the proofs above more carefully, it turns out that we have given a constructive existence proof of a solution $u(y, t)$ of Problem (I) and of its interfaces $\zeta_{i}(t)$. We mention that the existence of a finite time $t_{i}^{*}$ and the strict monotonicity of $\zeta_{i}(t)$ for $t>t_{i}^{*}$ could also be proved directly from the above construction. Only at one point did we really need the existing literature about porous media equations; namely, to prove the last statement of Theorem 4.2, we used the fact that $\zeta_{i} \in C^{1}\left(t_{i}^{*}, \infty\right)$.

5. Problem $\left(\mathrm{II}_{\mathrm{h}}\right)$ : Proof of Lemma 4.4. As we observed already in Sec. 3, Lemma 4.4 is nothing but the discretized version of (4.5), which was an immediate consequence of Lemma 4.3. Two ingredients were important in the proof of Lemma 4.3

(i) the fact that $\{v(t)\}^{1-\alpha}$ is uniformly bounded in $L^{1}\left(0, \frac{1}{2} L\right)$ for $0 \leq t \leq T$;

(ii) integration of the formula

$$
\frac{1}{1-\alpha}\left(v^{1-\alpha}\right)_{t}=v_{x x} .
$$

It is therefore not surprising that we shall need similar ingredients below with $v$ replaced by $v^{h}$. The first one is dealt with in the following Lemma.

Everywhere in this section we shall assume that $T>0$ and $1<\alpha^{\circ}<2$, that $v_{0}$ satisfies $\mathrm{H} 1-2$, that the mesh conditions (3.4) and (4.3) are satisfied, and that $v_{k}^{n}$ (or $\left.v^{h}(x, t)\right)$ is the solution of Problem $\left(\mathrm{II}_{\mathrm{h}}\right)$.

LEMma 5.1. There exists a function $f \in C\left(\left[0, \frac{1}{2} L\right]\right)$ such that

$$
v_{k}^{n} \geq f\left(x_{k}^{n}\right)>0 \text { if } 0<x_{k} \leq \frac{1}{2} L, 0 \leq t_{n} \leq T,
$$

and for all $\varepsilon>0$ there exists a $\delta>0$ which does not depend on $h$ such that

$$
\sum_{k=1}^{k_{0}}\left\{f\left(x_{k}\right)\right\}^{1-\alpha} \frac{1}{2}\left(\Delta x_{k}+\Delta x_{k+1}\right)<\varepsilon \quad \text { if } 0<x_{k_{0}}<\delta .
$$

The proof is based on the following comparison principle for Problem $\left(\mathrm{II}_{\mathrm{h}}\right)$.

Lemma 5.2. (Comparison Principle) Let for all $k=K_{0}+1, \ldots, K_{1}-1$ and $n=$ $0,1, \ldots, N$

$$
\left\{\begin{array}{l}
\frac{u_{k}^{n+1}-u_{k}^{n}}{\Delta t}-\left(u_{k}^{n}\right)^{\alpha} A u_{k}^{n+1} \leq \frac{v_{k}^{n+1}-v_{k}^{n}}{\Delta t}-\left(v_{k}^{n}\right)^{\alpha} A v_{k}^{n+1} \\
u_{K_{0}}^{n} \leq v_{K_{0}}^{n} \text { and } v_{K_{1}}^{n} \leq v_{K_{1}}^{n} \\
u_{k}^{0} \leq v_{k}^{0} .
\end{array}\right.
$$

Let (3.4) be satisfied with $\gamma$ satisfying

$$
\left|\frac{u_{k+1}^{n}-u_{k}^{n}}{x_{k+1}-x_{k}}\right| \leq \gamma \quad \text { and } \quad\left|\frac{v_{k+1}^{n}-v_{k}^{n}}{x_{k+1}-x_{k}}\right| \leq \gamma \quad \text { for }\left\{\begin{array}{l}
k=K_{0}, \ldots, K_{1}-1 \\
n=0, \ldots, N
\end{array}\right.
$$


Then

$$
u_{k}^{n} \leq v_{k}^{n} \quad \text { for }\left\{\begin{array}{l}
k=K_{0}, \ldots, K_{1} \\
n=0, \ldots, N .
\end{array}\right.
$$

Proof. We use induction with respect to $n$, and assume that for some $n=$ $0, \ldots, N-1$

$$
u_{k}^{n}-v_{k}^{n} \leq 0 \text { for all } k .
$$

Let $u_{k}^{n+1}-v_{k}^{n+1}$ be maximal with respect to $k$. Then

$$
\begin{aligned}
u_{k}^{n+1}-v_{k}^{n+1} \leq & u_{k}^{n}-v_{k}^{n}+\Delta t\left\{\left(u_{k}^{n}\right)^{\alpha} A u_{k}^{n+1}-\left(v_{k}^{n}\right)^{\alpha} A v_{k}^{n+1}\right\} \\
= & \Delta t\left(u_{k}^{n}\right)^{\alpha} A\left(u_{k}^{n+1}-v_{k}^{n+1}\right) \\
& +\left(u_{k}^{n}-v_{k}^{n}\right)\left\{1+\Delta t A v_{k}^{n+1} \frac{\left(u_{k}^{n}\right)^{\alpha}-\left(v_{k}^{n}\right)^{\alpha}}{u_{k}^{n}-v_{k}^{n}}\right\} \\
\leq & \left(u_{k}^{n}-v_{k}^{n}\right)\left\{1+\Delta t A v_{k}^{n+1} \frac{\left(u_{k}^{n}\right)^{\alpha}-\left(v_{k}^{n}\right)^{\alpha}}{u_{k}^{n}-v_{k}^{n}}\right\} \leq 0
\end{aligned}
$$

provided that

$$
\Delta t A v_{k}^{n+1} \frac{\left(u_{k}^{n}\right)^{\alpha}-\left(v_{k}^{n}\right)^{\alpha}}{u_{k}^{n}-v_{k}^{n}} \geq-1
$$

If

$$
A v_{k}^{n+1} \geq 0
$$

this is clearly satisfied. Otherwise we use the fact that

$$
0 \leq \frac{\left(u_{k}^{n}\right)^{\alpha}-\left(v_{k}^{n}\right)^{\alpha}}{u_{k}^{n}-v_{k}^{n}} \leq \alpha\left(v_{k}^{n}\right)^{\alpha-1} \leq \alpha \gamma^{\alpha-1} \min \left\{x_{k}^{\alpha-1},\left(L-x_{k}\right)^{\alpha-1}\right\}
$$

and hence, by (3.4),

$$
\Delta t\left|A v_{k}^{n+1}\right| \frac{\left(u_{k}^{n}\right)^{\alpha}-\left(v_{k}^{n}\right)^{\alpha}}{u_{k}^{n}-v_{k}^{n}} \leq \frac{2 \alpha \gamma^{\alpha} \Delta t \min \left\{x_{k}^{\alpha-1},\left(L-x_{k}\right)^{\alpha-1}\right\}}{\left(\Delta x_{k}+\Delta x_{k+1}\right) / 2} \leq 1 .
$$

REMARK. From the proof it follows that we do not need the mesh condition (3.4) if $A v_{k}^{n+1} \geq 0$, i.e., if $v$ is convex with respect to $x$. It is also easy to see that if only $u$ is convex with respect to $x$ we do not need (3.4).

Proof of Lemma 5.1. By hypothesis H2, either

$$
v_{0}(x) \geq A x \quad \text { on }\left[0, \frac{1}{2} L\right]
$$

for some $A>0$, or $v_{0}$ is convex near $x=0$. In the latter case we may assume without loss of generality that

$$
v_{0} \text { is convex on }\left[0, \frac{1}{2} L\right] \text {. }
$$

We choose $K_{1}$ such that $x_{K_{1}-1}<\frac{1}{2} L \leq x_{K_{1}}$. Since $v_{K_{1}}^{n}$ is uniformly bounded away from zero, there exists an $a \in(0,1]$ such that

$$
v_{K_{1}}^{n} \geq f\left(x_{k_{1}}\right) \quad 0 \leq n \leq N
$$

where

$$
f(x)= \begin{cases}a A x & \text { if }(5.3) \text { holds } \\ a v_{0}(x) & \text { if }(5.4) \text { holds }\end{cases}
$$


Since $f(x)$ is convex we may apply Lemma 5.2 with $u_{k}^{n}=f\left(x_{k}\right)$ and we conclude that

$$
v_{k}^{n} \geq f\left(x_{k}\right) \quad 0 \leq k \leq K_{1}, 0 \leq n \leq N .
$$

Since $f$ is a nondecreasing function on $\left[0, \frac{1}{2} L\right]$,

$$
\sum_{k=1}^{k_{0}}\left\{f\left(x_{k}\right)\right\}^{1-\alpha} \Delta x_{k} \leq \int_{0}^{x_{k_{0}}} f^{1-\alpha}(x) d x .
$$

By (4.3),

$$
\frac{1}{2}\left(\Delta x_{k}+\Delta x_{k+1}\right) \leq \frac{1}{2}\left(A_{2}+1\right) \Delta x_{k}
$$

and (5.2) follows from the fact that $f^{1-\alpha} \in L^{1}\left(0, \frac{1}{2} L\right)$.

The second ingredient we needed was a discretized version of Eq. (5.1). Here the difficulty is that at a discretized level Eq. (5.1) and the original equation $v_{t}=v^{\alpha} v_{x x}$ are no longer equivalent.

LEMMA 5.3. If we define

$$
I_{k}^{n}=\frac{1}{(\alpha-1) \Delta t}\left\{\left(v_{k}^{n+1}\right)^{1-\alpha}-\left(v_{k}^{n}\right)^{1-\alpha}\right\},
$$

then, for all $0 \leq n \leq N-1,1 \leq k \leq K-1$,

$$
0 \leq I_{k}^{n}+A v_{k}^{n+1} \leq A v_{k}^{n+1}\left\{1-\left(\frac{v_{k}^{n}}{v_{k}^{n+1}}\right)^{\alpha}\right\} .
$$

Proof. Let $\phi \in C^{1}(I)$ be a convex function on an interval $I$. Then for all $a, b \in I$

$$
\phi^{\prime}(a)(b-a) \leq \phi(b)-\phi(a) \leq \phi^{\prime}(b)(b-a) .
$$

Applying (5.5) to the convex function

$$
\phi(s)=\frac{1}{\alpha-1} s^{1-\alpha} \quad s>0
$$

we find that

$$
-\left(v_{k}^{n}\right)^{-\alpha}\left(v_{k}^{n+1}-v_{k}^{n}\right) \leq I_{k}^{n} \Delta t \leq-\left(v_{k}^{n+1}\right)^{-\alpha}\left(v_{k}^{n+1}-v_{k}^{n}\right) .
$$

Thus

$$
\begin{aligned}
0 & =-\left(v_{k}^{n}\right)^{-\alpha} \frac{v_{k}^{n+1}-v_{k}^{n}}{\Delta t}+A v_{k}^{n+1} \leq I_{k}^{n}+A v_{k}^{n+1} \\
& \leq-\left(v_{k}^{n+1}\right)^{-\alpha} \frac{v_{k}^{n+1}-v_{k}^{n}}{\Delta t}+A v_{k}^{n+1}=A v_{k}^{n+1}\left\{1-\left(\frac{v_{k}^{n}}{v_{k}^{n+1}}\right)^{\alpha}\right\} .
\end{aligned}
$$

Proof of Lemma 4.4. Let $\varepsilon>0$. Since $\left|w_{k}^{n}\right| \leq \gamma$, it is sufficient to prove that for all $0<\tau<T$ there exists a $\delta>0$ such that for all small values of $|h|$

$$
\left|\sum_{n=n_{0}+1}^{n_{1}}\left(w_{k_{0}}^{n}-w_{1}^{n}\right) \Delta t\right|<\varepsilon \quad \text { if } 0<x_{k_{0}}<\delta, \tau \leq t_{n_{0}+1} \leq t_{n_{1}} \leq T,
$$


or, replacing $n$ by $n+1$ and using the definition of $A v_{k}^{n+1}$, that

$$
J_{k_{0}}^{n_{0}, n_{1}} \equiv\left|\sum_{n=n_{0}}^{n_{1}-1} \sum_{k=1}^{k_{0}-1} A v_{k}^{n+1} \frac{1}{2}\left(\Delta x_{k}+\Delta x_{k+1}\right) \Delta t\right|<\varepsilon
$$

if $\tau \leq t_{n_{0}+1} \leq t_{n_{1}} \leq T$ and $0<x_{k_{0}}<\delta$.

We fix $\tau \in(0, T)$. By Lemma 5.3

$$
J_{k_{0}}^{n_{0}, n_{1}} \leq J_{1}+J_{2}
$$

where

$$
J_{1}=\left|\sum_{n=n_{0}}^{n_{1}-1} \sum_{k=1}^{k_{0}-1} I_{k}^{n} \frac{1}{2}\left(\Delta x_{k}+\Delta x_{k+1}\right) \Delta t\right|
$$

and

$$
J_{2}=\sum_{n=n_{0}}^{n_{1}-1} \sum_{k=1}^{k_{0}-1} A_{v_{k}}^{n+1}\left\{1-\left(\frac{v_{k}^{n}}{v_{k}^{n+1}}\right)^{\alpha}\right\} \frac{1}{2}\left(\Delta x_{k}+\Delta x_{k+1}\right) \Delta t .
$$

Here we observe that all terms of $J_{2}$ are nonnegative.

Since

$$
J_{1}=\frac{1}{\alpha-1}\left|\sum_{k=1}^{k_{0}-1}\left\{\left(v_{k}^{n_{1}}\right)^{1-\alpha}-\left(v_{k}^{n_{0}}\right)^{1-\alpha}\right\} \frac{1}{2}\left(\Delta x_{k}+\Delta x_{k+1}\right)\right|,
$$

it follows from Lemma 5.1 that for $0<x_{k_{0}}<\delta$

$$
J_{1} \leq \frac{2}{\alpha-1} \sum_{k=1}^{k_{0}-1}\left\{f\left(x_{k}\right)\right\}^{1-\alpha} \frac{1}{2}\left(\Delta x_{k}+\Delta x_{k+1}\right)<\frac{1}{2} \varepsilon
$$

if we choose $\delta$ small enough.

We rewrite

$$
J_{2}=\sum_{n=n_{0}}^{n_{1}-1} \sum_{k=1}^{k_{0}-1}\left(w_{k+1}^{n+1}-w_{k}^{n+1}\right)\left(v_{k}^{n+1}\right)^{1-\alpha} \frac{\left(v_{k}^{n+1}\right)^{\alpha}-\left(v_{k}^{n}\right)^{\alpha}}{v_{k}^{n+1}} \Delta t .
$$

Using that $w_{k+1}^{n+1}-w_{k}^{n+1} \leq 2 \gamma$, we obtain from Lemma 5.1 and the mesh condition (3.4) that

$$
J_{2} \leq \alpha^{-1} \gamma^{1-\alpha}\left\{\max _{1 \leq k \leq k_{0}-1} x_{k}^{1-\alpha} \sum_{n=n_{0}}^{n_{1}-1}\left|Q_{k}^{n}\right| \Delta t\right\} \sum_{k=1}^{k_{0}-1}\left\{f\left(x_{k}\right)\right\}^{1-\alpha} \frac{1}{2}\left\{\Delta x_{k}+\Delta x_{k+1}\right\},
$$

where we have written

$$
Q_{k}^{n}=\frac{\left(v_{k}^{n+1}\right)^{\alpha}-\left(v_{k}^{n}\right)^{\alpha}}{v_{k}^{n+1} \Delta t}
$$

If we can prove that for some $\delta_{0}>0$ and $C>0$

$$
x_{k}^{1-\alpha} \sum_{n=n_{0}}^{n_{1}-1}\left|Q_{k}^{n}\right| \Delta t \leq C \quad \text { if } 0<x_{k} \leq \delta_{0},
$$


we can choose, in view of Lemma $5.1, \delta \in\left(0, \delta_{0}\right)$ so small that

$$
J_{2} \leq \alpha^{-1} \gamma^{1-\alpha} C \sum_{k=1}^{k_{0}-1}\left\{f\left(x_{k}\right)\right\}^{1-\alpha} \frac{1}{2}\left\{\Delta x_{k}+\Delta x_{k+1}\right\}<\frac{1}{2} \varepsilon
$$

and (5.6) follows.

So it remains to prove (5.7). We write $\left|Q_{k}^{n}\right|=\left\{Q_{k}^{n}\right\}^{+}+\left\{Q_{k}^{n}\right\}^{-}$and estimate first the terms with $\left\{Q_{k}^{n}\right\}^{-}$.

Since $\Delta t \leq|h|<h_{0}$, it follows from Lemma 3.2 that

$$
\frac{v_{k}^{n}}{v_{k}^{n+1}}=\left\{1+\frac{v_{k}^{n+1}-v_{k}^{n}}{v_{k}^{n}}\right\}^{-1} \leq\left(1-\frac{h_{0}}{\alpha \tau}\right)^{-1} \leq C_{1} \text { for } t_{n+1} \geq \tau
$$

if we choose $0<h_{0}<\alpha \tau$. Hence, using Lemma 3.2 again,

$$
\begin{aligned}
\sum_{n=n_{0}}^{n_{1}-1}\left\{Q_{k}^{n}\right\}^{-} \Delta t & \leq T \max _{n_{0} \leq n \leq n_{1}-1}\left\{Q_{k}^{n}\right\}^{-} \\
& =T \max _{n_{0} \leq n \leq n_{1}-1} \frac{\left\{v_{k}^{n+1}-v_{k}^{n}\right\}^{-}}{v_{k}^{n} \Delta t} \cdot \frac{\left\{\left(v_{k}^{n}\right)^{\alpha}-\left(v_{k}^{n+1}\right)^{\alpha}\right\}^{+}}{\left\{v_{k}^{n}-v_{k}^{n+1}\right\}^{+}} \cdot \frac{v_{k}^{n}}{v_{k}^{n+1}} \\
& \leq T C_{1} \tau^{-1} \max _{n_{0} \leq n \leq n_{1}-1}\left(v_{k}^{n}\right)^{\alpha-1} \leq T C_{1} \tau^{-1} \gamma^{\alpha-1} x_{k}^{\alpha-1},
\end{aligned}
$$

where we have used the fact that, in view of the convexity of the function $g(s)=s^{\alpha}$ and (5.5),

$$
\left(v_{k}^{n}\right)^{\alpha}-\left(v_{k}^{n+1}\right)^{\alpha} \leq \alpha\left(v_{k}^{n}\right)^{\alpha-1}\left\{v_{k}^{n}-v_{k}^{n+1}\right\} .
$$

Finally we estimate the terms with $\left\{Q_{k}^{n}\right\}^{+}$. Since the function $g(s)=s^{\alpha /(\alpha-1)}=$ $s^{1+1 /(\alpha-1)}$ is convex for $s>0$, it follows from (5.5) that

$$
\begin{aligned}
\left(v_{k}^{n+1}\right)^{\alpha}-\left(v_{k}^{n}\right)^{\alpha} & =g\left(\left(v_{k}^{n+1}\right)^{\alpha-1}\right)-g\left(\left(v_{k}^{n}\right)^{\alpha-1}\right) \\
& \leq \frac{\alpha-1}{\alpha}\left\{\left(v_{k}^{n+1}\right)^{\alpha-1}-\left(v_{k}^{n}\right)^{\alpha-1}\right\} v_{k}^{n+1},
\end{aligned}
$$

and thus

$$
\left\{Q_{k}^{n}\right\}^{+} \leq \frac{\alpha-1}{\alpha} \frac{1}{\Delta t}\left\{\left(v_{k}^{n+1}\right)^{\alpha-1}-\left(v_{k}^{n}\right)^{\alpha-1}\right\}^{+}
$$

Since $a^{+}=a+a^{-}(a \in \mathbf{R})$

$$
\frac{\alpha}{\alpha-1} \sum_{n=n_{0}}^{n_{1}-1}\left\{Q_{k}^{n}\right\}^{+} \Delta t \leq\left(v_{k}^{n_{1}}\right)^{\alpha-1}-\left(v_{k}^{n_{0}}\right)^{\alpha-1}+\sum_{n=n_{0}}^{n_{1}-1}\left\{\left(v_{k}^{n}\right)^{\alpha-1}-\left(v_{k}^{n+1}\right)^{\alpha-1}\right\}^{+} .
$$

Because $1<\alpha<2$, the function $g(s)=s^{\alpha-1}$ is concave for $s>0$ and thus

$$
\left(v_{k}^{n}\right)^{\alpha-1}-\left(v_{k}^{n+1}\right)^{\alpha-1} \leq(\alpha-1)\left(v_{k}^{n+1}\right)^{\alpha-2}\left(v_{k}^{n}-v_{k}^{n+1}\right) .
$$


Hence

$$
\begin{aligned}
\sum_{n=n_{0}}^{n_{1}}\left\{Q_{k}^{n}\right\}^{+} \Delta t & \leq \frac{\alpha}{\alpha-1}\left(v_{k}^{n_{1}}\right)^{\alpha-1}+\alpha \sum_{n=n_{0}}^{n_{1}-1}\left(v_{k}^{n+1}\right)^{\alpha-2}\left\{v_{k}^{n}-v_{k}^{n+1}\right\}^{+} \\
& \leq x_{k}^{\alpha-1} \alpha \gamma^{\alpha-1}\left\{\frac{1}{\alpha-1}+T \max _{n_{0} \leq n \leq n_{1}-1} \frac{\left\{v_{k}^{n+1}-v_{k}^{n}\right\}^{-}}{v_{k}^{n_{1}+1} \Delta t}\right\} \\
& \leq x_{k}^{\alpha-1} \alpha \gamma^{\alpha-1}\left\{\frac{1}{\alpha-1}+\frac{C_{1} T}{\alpha \tau}\right\}
\end{aligned}
$$

where we used Lemma 3.2 and (5.8) in the last inequality. Combining this with (5.9) we arrive at (5.7), and the proof is complete.

6. Numerical simulations. In this section we discuss some computational results. All computations were made on a VAX 750 .

First we test the scheme using the fact that Problem (I) has an explicit solution, the so-called Barenblatt-Pattle solution. If $m=2$, it is given by

$$
u(y, t)= \begin{cases}\frac{1}{\zeta(t)}\left\{1-\left(\frac{y}{\zeta(t)}\right)^{2}\right\} & \text { if }|y| \leq \zeta(t) \\ 0 & \text { if }|y|>\zeta(t)\end{cases}
$$

where the interfaces $y= \pm \zeta(t)$ are defined by

$$
\zeta(t)=\{12(t+1)\}^{1 / 3} \text { for } t \geq 0 .
$$

In Fig. 1 we compare the exact and numerical interfaces.

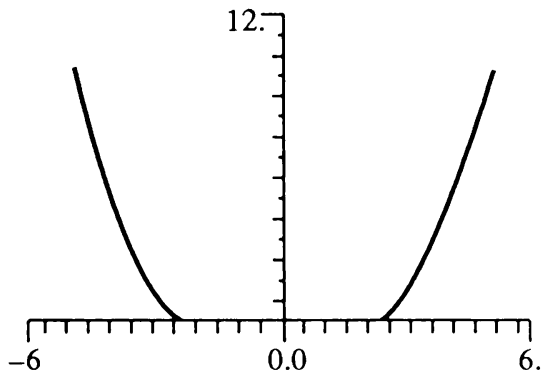

a. The exact interfaces.

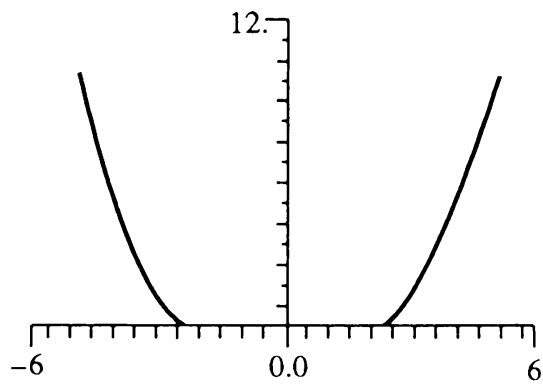

b. The numerical interfaces (with 39 mesh points).

FIG. 1. The Barenblatt-Pattle solution $(m=2)$.

As a second example we consider Problem (I), still with $m=2$, with initial function

$$
u_{0}(y)= \begin{cases}\frac{1}{2}(1-\theta) \sin ^{2} y+\frac{1}{2} \theta \sin ^{4} y & \text { if }-\pi<y<0 \\ 0 & \text { otherwise }\end{cases}
$$

where we choose $\theta$ respectively equal to $0, \frac{1}{2}$, and 1 . In this case the waiting-time $t^{*}$, which we defined in the introduction, is strictly positive and it follows from results 
by Aronson, Caffarelli, and Kamin [3] that

$$
\begin{aligned}
& \theta=0 \Rightarrow t^{*}=\frac{1}{6} \\
& \theta=\frac{1}{2} \Rightarrow \frac{1}{6}<t^{*} \leq \frac{1}{3} \\
& \theta=1 \Rightarrow \frac{19}{60} \leq t^{*}<\infty .
\end{aligned}
$$

In addition is was shown in [3] that, if $\zeta$ denotes the right interface

$$
\theta=0 \Rightarrow \lim _{t \backslash t^{*}} \zeta^{\prime}(t)=0
$$

and, in [4], that

$$
\theta=1 \Rightarrow \lim _{t \searrow t^{*}} \zeta^{\prime}(t)>0
$$

If $\theta=\frac{1}{2}$, this problem is open.

The numerical interfaces are shown in Fig. 2.

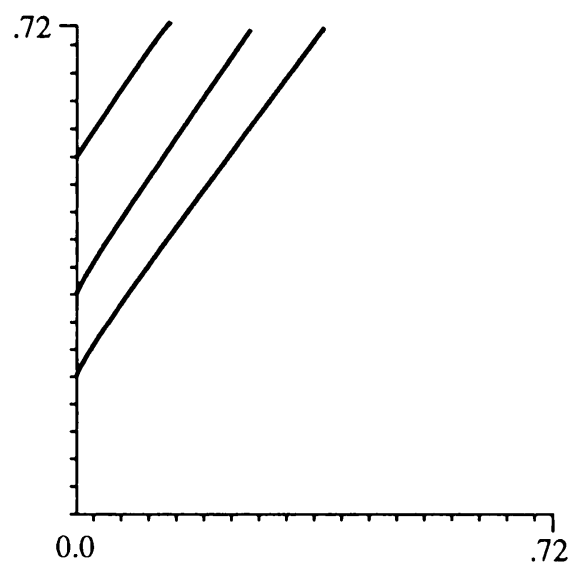

FIG. 2. The numerical right interfaces with $m=2$ and initial function (6.1) (with 99 mesh points).

Observe that the results are in correspondence with the properties described above. In addition the numerical interfaces are very close to the ones computed by Mimura, Nakaki, and Tomoeda [16].

A clear advantage of our scheme is its simple structure with the consequence that the computing time is always very short. Nevertheless it gives us accurate results; also this scheme does not contain an artificial viscosity term. We have observed from numerical experiments that, for the fast convergence of the scheme, mesh conditions like (3.4) and (4.3) are crucial.

The simplicity of the scheme is of course due to the fact that the quantity $\int_{-\infty}^{\infty} u(y, t) d y=L$ does not depend on $t$, which reduces the free-boundary problem for $u(y, t)$ to a problem for $v(x, t)$ with fixed boundaries. We lose this property if we want to solve, for example, equations of the type

$$
u_{t}=\left(u^{m}\right)_{y y}+f(u)
$$


with $f(0)=0$, which could be treated by means of a splitting strategy (see [13, $16])$, but in that case we have to use the inverse transformation to compute $L\left(t_{n}\right)=$ $\int_{-\infty}^{\infty} u\left(y, t_{n}\right) d y$.

Acknowledgment. We gratefully acknowledge a valuable discussion with M. E. Gurtin, who pointed out to us the importance of the transformation (1.3).

\section{REFERENCES}

[1] D. G. Aronson, Nonlinear diffusion problems, Free Boundary Problems: Theory and Applications, Vol. 1 (eds. A. Fasano and M. Primicerio), Research Notes in Mathematics 78, Pitman, London, 1983, pp. 134-149

[2] D. G. Aronson, The porous medium equation, Some Problems in Nonlinear Diffusion (eds. A. Fasano and M. Primicerio), Lecture Notes in Mathematics 1224 (CIME Foundation Series), Springer, Berlin, (1986)

[3] D. G. Aronson, L. A. Caffarelli, and S. Kamin, How an initially stationary interface begins to move in porous medium flow, SIAM J. Math. Anal. 14, 639-658 (1983)

[4] D. G. Aronson, L. A. Caffarelli, and J. L. Vazquez, Interfaces with a corner point in one-dimensional porous medium flow, Comm. Pure Appl. Math. 38, 375-404 (1985)

[5] V. F. Baklanovskaja, The numerical solution of a one-dimensional problem for equations of nonstationary filtrations, Zh. Vychisl. Mat. i Mat. Fiz. 1, 461-469 (1961)

[6] A. E. Berger, H. Brézis, and J. C. W. Rogers, A numerical method for solving the problem $u_{t}-\Delta f(u)=$ 0, RAIRO Anal. Num. 13, 297-312 (1979)

[7] J. G. Berryman, Slow diffusion on the line, J. Math. Phys. 21, 1326-1331 (1980)

[8] M. Bertsch, R. Dal Passo, and M. Ughi, Nonuniqueness of solutions of a degenerate parabolic equation, preprint no. 4, Ist. Appl. Calc., Rome (1988)

[9] M. Bertsch, C. J. Van Duyn, J. R. Esteban, and Zhang Hongfei, Regularity of the free boundary in a doubly degenerate parabolic equation, Comm. Partial Differential Equations 14, 391-412 (1989)

[10] L. A. Caffarelli and A. Friedman, Regularity of the free boundary for the one-dimensional flow of gas in a porous medium, Amer. J. Math. 101, 1193-1281 (1979)

[11] R. Dal Passo and S. Luckhaus, On a degenerate diffusion problem not in divergence form, to appear in J. Differential Equations 69, 1-14 (1987)

[12] E. DiBenedetto and D. Hoff, An interface tracking algorithm for the porous medium equation, Trans. Amer. Math. Soc. 288, 463-500 (1984)

[13] J. L. Graveleau and P. Jamet, A finite difference approach to some degenerate nonlinear parabolic equations, SIAM J. Appl. Math. 20, 199-223 (1971)

[14] M. E. Gurtin, R. C. MacCamy, and E. A. Socolovsky, A coordinate transformation for the porous media equation that renders the free-boundary stationary, Quart. Appl. Math. 42, 345-357 (1984)

[15] D. Hoff, A linearly implicit finite difference scheme for the one-dimensional porous medium equation, Math. Comp. 45, 23-33 (1985)

[16] M. Mimura, T. Nakaki, and K. Tomoeda, A numerical approach to interface curves for some nonlinear diffusion equations, Japan J. Appl. Math. 1, 93-139 (1984)

[17] M. Mimura and K. Tomoeda, Numerical approximations to interface curves for a porous media equation, Hiroshima Math. J. 13, 273-294 (1983)

[18] M. E. Rose, Numerical methods for flows through porous media-I, Math. Comp. 40, 435-467 (1983)

[19] M. E. Rose, Numerical methods for a porous medium equation, Argonne Nat. Lab. Rep., Argonne, Illinois (1978)

[20] M. E. Rose, Numerical methods for a general class of porous medium equations, Argonne Nat. Lab. Rep., Argonne, Illinois (1980)

[21] G. Rosen, Nonlinear heat conduction in solid $\mathrm{H}_{2}$, Phys. Rev. B 19, 2398-2399 (1979)

[22] E. A. Socolovsky, Difference schemes for degenerate nonlinear parabolic equations, preprint CarnegieMellon Univ., Pittsburgh (1983)

[23] M. Ughi, A degenerate parabolic equation modelling the spread of an epidemic, Ann. Mat. Pura Appl. 143, 385-400 (1986)

[24] J. L. Vazquez, The interface of one-dimensional flows in porous media, Trans. Amer. Math. Soc. 285, 717-737 (1984) 